\title{
Structure and Correlations of the Flux Line Lattice in Crystalline Nb through the Peak Effect
}

\author{
P. L. Gammel, U. Yaron,* A. P. Ramirez, D. J. Bishop, A. M. Chang, ${ }^{\dagger}$ R. Ruel, L. N. Pfeiffer, and E. Bucher \\ Bell Labs, Lucent Technologies, 700 Mountain Avenue, Murray Hill, New Jersey 07974 \\ G. D’Anna \\ Institut de Génie Atomique, Ecole Polytechnique Fédérale de Lausanne, CH-1015 Lausanne, Switzerland \\ D. A. Huse \\ Princeton University, Princeton, New Jersey \\ K. Mortensen and M. R. Eskildsen \\ Risø National Laboratory, DK-4000 Roskilde, Denmark \\ P. H. Kes \\ Leiden University, Kamerlingh Onnes Laboratory, P.O. Box 9506, 2300 RA Leiden, The Netherlands
}

(Received 15 August 1997)

\begin{abstract}
We have measured the structure of the field cooled flux line lattice (FLL) in single crystal $\mathrm{Nb}$ using small angle neutron scattering. Augmented by transport and thermodynamic data, a scenario for the dramatic disordering of the FLL near the peak effect emerges. A precursor to the peak effect is an hexatic FLL as positional order is lost. As the critical current rises, the orientational and longitudinal correlation lengths also fall, leading to an amorphous array of lines at the critical current maximum. [S0031-9007(97)05112-0]
\end{abstract}

PACS numbers: 74.60.Ec, 74.25.Dw, 74.60.Ge, 74.60.Jg

A reexamination of the statics and dynamics of the flux line lattice (FLL) in conventional materials is now taking place [1], focusing on the peak effect in the critical current, a violent maelstrom of disordered flux motion interacting with pinning defects. Although this effort was stimulated by the new understanding of the FLL phase diagram [2] in high temperature and thin film superconductors, the peak effect has become central to understanding the unusual dynamic response of the FLL. The nature of the peak effect near FLL phase transitions in the high- $T_{c}$ superconductors, while probably different in detail, is similarly a topic of intense scrutiny [3].

In this Letter we present structural data, using small angle neutron scattering (SANS), on the FLL of single crystal $\mathrm{Nb}$ through the regime of the peak effect. We present the first measurements of all three FLL correlation lengths, two transverse and one longitudinal. These data, combined with thermodynamic and transport studies on the same sample, lead to a comprehensive model of the morphology for the field cooled FLL through the peak effect.

Our study was performed on a cylindrical, $6.7 \mathrm{~mm} \times$ $\varnothing=4 \mathrm{~mm}$, annealed $\mathrm{Nb}$ crystal. The [110] axis was parallel to within $5^{\circ}$ of the cylinder axis. The crystal mosaic was $0.2^{\circ}$, with no signs of additional domains in bright field diffraction images. This was the best mosaic obtained during annealing studies, and it was also our largest monodomain sample. Transport showed a residual resistivity ratio $\approx 1000, T_{c}=9.16 \mathrm{~K}$ and a superconducting transition width less than $40 \mathrm{mK}$. Magnetization and specific heat data were consistent with earlier studies of high purity $\mathrm{Nb}$ [4]. Of particular relevance to the SANS data is the value of $\kappa_{1}=H_{c 2} / \sqrt{2} H_{c}=0.96 \lambda_{L}(0) / \xi_{0}$. At $T=6.4 \mathrm{~K}, \kappa_{1}=0.99$, and at $T=4.6, \kappa_{1}=1.24$. These values attest to the high quality of our sample, and also point to its proximity to the type-I-type-II boundary, which limits the low field range of our data.

Our studies were performed at the SANS facility in the cold neutron guide hall of the Ris $\varnothing$ DR3 reactor. The crystal was masked with cadmium, leaving a circular hole $3.7 \mathrm{~mm}$ in diameter to reduce specular reflections and scattering from the distorted FLL near the sample surface. The magnetic field was parallel to both the incident neutrons and the cylinder axis of the sample. The angular divergence, which dominated the experimental resolution, was between $0.13^{\circ}$ and $0.18^{\circ}$ FWHM. The energy divergence was fixed at $\Delta \lambda / \lambda=0.18$. The diffracted neutrons were counted by an area detector located $6 \mathrm{~m}$ from the sample.

The FLL for this orientation is nearly hexagonal and oriented by the crystal lattice [5]. Shown in Fig. 1 are the average $\mathbf{q}$ and reflectivity, extracted from integrated intensity over rocking curves, for the first order diffraction peaks. The low field region of constant $\mathbf{q}$ and falling intensities [6] is the intermediate mixed state, typical of marginally type-II, clean samples. We concentrate our analysis on $h=H / H_{c 2}>0.65$, where the sample is fully in the mixed state.

At the intensity peak, the reflectivity into the first order diffraction pattern approaches $10 \%$, and all orders of 


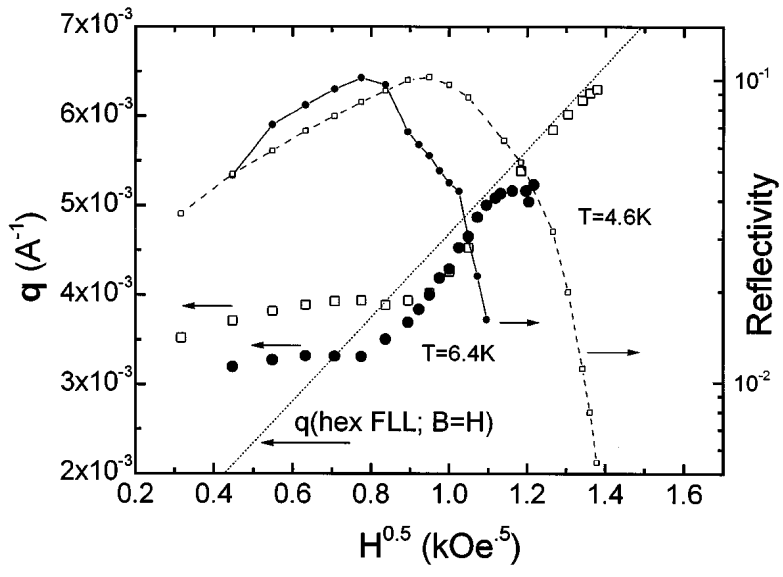

FIG. 1. Shown are the $q$ value and reflectivity of the first diffraction peak at $T=4.6 \mathrm{~K}$ (open symbols, dashed line) and $6.4 \mathrm{~K}$ (solid symbols, full line) versus field. For $h=H / H_{c 2}<$ 0.5 , the sample is in the intermediate mixed state. Analysis of the SANS data was performed for $h>0.65$, where the sample is in the mixed state.

diffraction out to $(3,0)$ are seen. Such large reflectivity leads to substantial multiple scattering corrections for higher order reflections, so our analysis is derived entirely from the first order peaks. Analysis of the form factor was performed using first order Ginzburg-Landau (GL) corrections to the London model [7]. Separate fits using the Clem model [8] gave $\kappa_{1}(T=6.4 \mathrm{~K})=0.82$ and $\kappa_{1}(T=4.6 \mathrm{~K})=1.34$, consistent with the GL analysis and in agreement with thermodynamic studies. Typical of these models, the individual values of $\xi=29.7 \mathrm{~nm}$ and $\lambda=25.2 \mathrm{~nm}$ are both about $30 \%$ lower than the thermodynamics data.

Three FLL correlation lengths can be determined [9] from a SANS experiment. Fits to the azimuthal and radial widths of data in the plane of the detector, such as are shown for three different fields in Fig. 2, give information on two correlation lengths perpendicular to the flux lines. The radial width, corrected for instrumental resolution, gives the positional correlation length $\xi^{\|}$, parallel to the reciprocal lattice vector $\mathbf{G}$, and is related to the compressive displacements of the FLL. Similarly, the azimuthal width gives $\xi^{\perp}$, perpendicular to $\mathbf{G}$, which is related to shear displacements. The last length, derived from the width of the rocking curve in our geometry, is the longitudinal correlation length $\xi^{L}$, a measure of the correlations along the flux lines, which is related to tilt displacements. Our experimental setup is optimized for $\xi^{L}$, where the resolution is roughly 2 orders of magnitude better than for either $\xi^{\|}$or $\xi^{\perp}$. Macroscopic field gradients, which can influence $\xi^{\|}$and $\xi^{\perp}$ were minimized using both a field cooled procedure and masking of the perimeter of the sample. In addition, such effects were checked for using analysis of higher order diffraction peaks and scans at different neutron wavelengths, and were negligible.

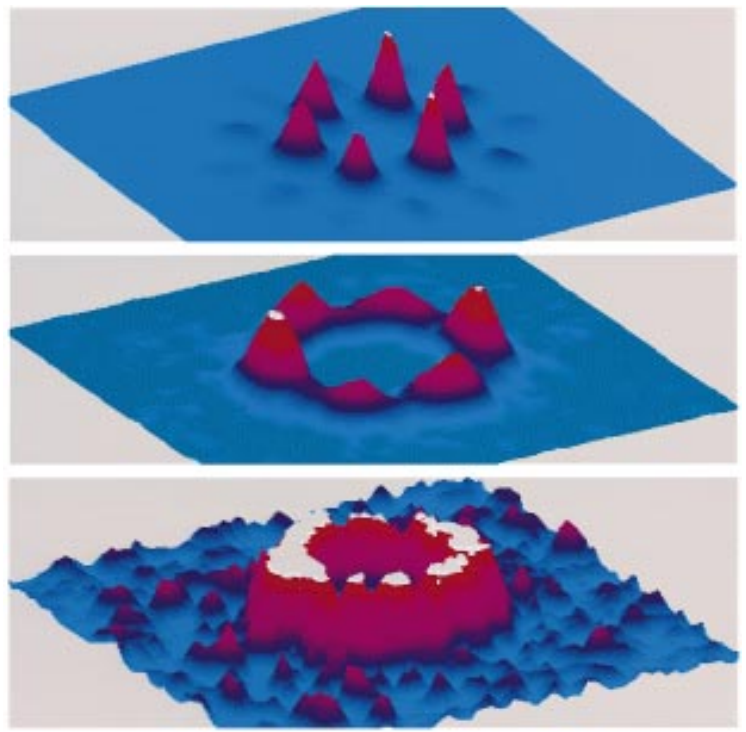

FIG. 2(color). Shown are diffraction patterns from the FLL in three regimes at $T=4.6 \mathrm{~K}$. Top: FLL crystal $(H=$ $1.1 \mathrm{kOe}, h=0.47)$. Middle: hexatic FLL just below the peak effect $(H=2.15, h=0.91)$. Bottom: ring of scattering from amorphous FLL $(H=2.25, h=0.96)$. The radial width of the peaks determines the positional correlation length $\left(\sim 1 a_{0}\right)$, the azimuthal width of the peaks gives the orientational correlation length, and rocking through the Bragg gives the longitudinal correlation length $\left(\sim 100 a_{0}\right)$. Nonuniform peak intensities are instrumental artifacts.

The results of the SANS experiments at $T=$ $6.4 \mathrm{~K}$ and $T=4.6 \mathrm{~K}$ are summarized in Fig. 3. The data are plotted versus reduced field $h=H / H_{c 2}$, where $H_{c 2}(T=6.4 \mathrm{~K})=1.55 \mathrm{kOe}$ and $H_{c 2}(T=4.6 \mathrm{~K})=$ $2.35 \mathrm{kOe}$. The positional correlation length, normalized to the measured FLL spacing $a_{0}(\mu \mathrm{m})=0.134 B^{-1 / 2}(\mathrm{kG})$, is shown in the middle panel. At low fields, all correlation lengths are long, and the FLL is a well ordered crystal as shown in Fig. 2, top. As a precursor to the peak effect, we find that $\xi^{\|} / a_{0} \sim 2$ at the minimum in the critical current, where strong deviations from $J_{c} h=$ const begin at both temperatures. As the critical current rises, we find $\xi^{\|} / a_{0} \rightarrow 1$ (Fig. 2, center). Commensurate with $\xi^{\|} / a_{0} \sim 2$ is the onset of a rapid reduction in $\xi^{\perp}$, shown in the bottom panel of Fig. 3. A uniform ring of scattering (Fig. 2, bottom), with $\xi^{\|} / a_{0}=1$ and $\xi^{\perp} / a_{0}=1$, is seen only at the peak in the critical current. This dramatic and complete positional disordering is in accord with the widely accepted scenario for the peak effect: It is a static, structural disordering of the FLL.

Pioneering studies of the positional correlation length were first performed by Lippmann et al. [10]. In those data, the focus was on the doping dependence, and the regime of the peak effect was not measured. More recently [11], Lynn et al. studied the two transverse correlation lengths $\xi^{\|}$and $\xi^{\perp}$, interpreting the reduction of these length scales in terms of FLL melting, although 


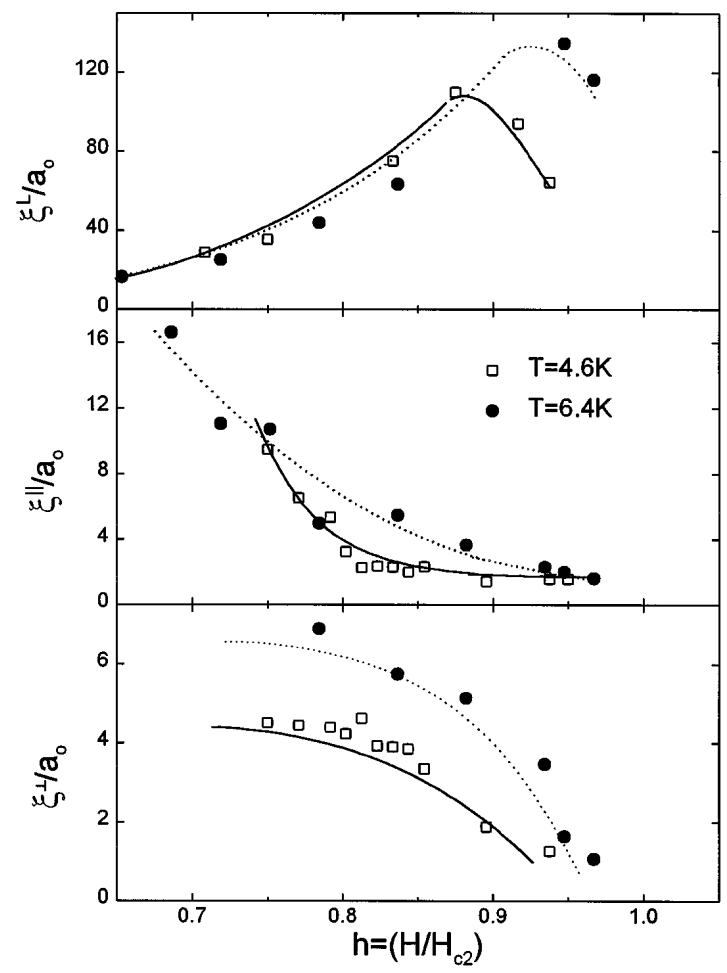

FIG. 3. Shown is a summary of all three correlation lengths extracted from the SANS data at $T=4.6 \mathrm{~K}$ (open squares) and $6.4 \mathrm{~K}$ (solid circles). The lines are guides to the eye.

the data in this case did not extend to the amorphous ring of scattering. In the range of overlap, our data for $\xi^{\|}$and $\xi^{\perp}$ are qualitatively similar to Lynn et al., although the lower $\kappa_{1}$ of our crystal shifts the field scale.

To complete these data, we also measured $\xi^{L}$. These data are shown in the top panel in Fig. 3. Somewhat surprisingly, up to the rise in the critical current, $\xi^{L}$ is large and increasing with field. Only above this rise, where the scattering in the plane of the detector is approaching an isotropic ring, do we see a reduction in the longitudinal correlations. Even at the peak in the critical current, however, $\xi^{L} / a_{0} \gg 1$, meaning that the flux lines are still extended objects.

We have attempted to fit the data below the peak effect using the Larkin-Ovchinnikov (LO) collective pinning model [12], which relates the correlation length to the measured critical current. This gave poor fits to the field dependence and also required unacceptable numerical prefactors $(\sim 0.01)$ in the expression for the correlation lengths. While this model has shown to work well at the low fields [13], it is generally accepted that it should break down near the peak effect. In the regime above the maximum in $\xi^{L} / a_{0}$, however, the amorphous or single vortex pinning limit [14] of the LO theory fits our data. This limit, appropriate for $\xi^{\|} / a_{0} \sim 1$, gives $\xi^{L}=A\left[\varepsilon_{0} a_{0}(1-h) / j_{c} \varnothing_{0}\right]^{1 / 2}$. Using $A \sim 0.7$, from our low field studies, gives quite reasonable fits at both temperatures.
In the regime where the amorphous limit of the LO theory describes our data, we can use the same approximation to understand the microscopic origin of the pinning. Defining $w=\xi^{L} \xi^{\perp 2} H_{c 2}\left(j_{c} h\right)^{2}=w_{0}(T) h^{p}(1-$ $h)^{2}$ gives $p=1$ for pinning due to variations in $T_{c}$ and $p=3$ for variations in mean free path. Our data are consistent with $p=1$, which seems plausible as $T_{c}$ in $\mathrm{Nb}$ is very sensitive to variations in local oxygen, hydrogen, or nitrogen concentration. The prefactor also agrees with the expected $w_{0}(T) \sim\left[1-\left(T / T_{c}\right)\right]^{2}$. Thus, the major pinning centers appear to be point defects due to such impurities.

In order to locate the FLL features precisely relative to the peak effect of this sample, we have measured the critical current using local magnetization, global magnetization, and low frequency transport. For magnetization studies, the sample was heat treated in oxygen for $10 \mathrm{~min}$ at $330{ }^{\circ} \mathrm{C}$ to reduce surface effects. This heat treatment, while leaving the peak effect virtually unaffected, reduced the hysteresis just below the peak by almost a factor of 4. The critical state model [15] was used to extract the critical currents shown in Fig. 4. Transport critical currents were measured on $0.35 \mathrm{~mm} \times 0.35 \mathrm{~mm} \times 3.5 \mathrm{~mm}$ bars cut from the large sample. These bars were etched and heat treated, and data were taken with the sample immersed in ${ }^{4} \mathrm{He}$ to reduce joule heating. The peak effect data are qualitatively similar to the magnetization critical currents, but were always contaminated by surface defects and damage near the contacts. In the magnetization critical currents, the peak effect increases in magnitude and shifts to slightly lower reduced field as the temperature is reduced. At lower temperatures in these clean samples, $\kappa_{1}$ increases and the sample is more strongly type-II. This agrees with our SANS data, where the disordering feature also moves to lower reduced field at lower temperature.

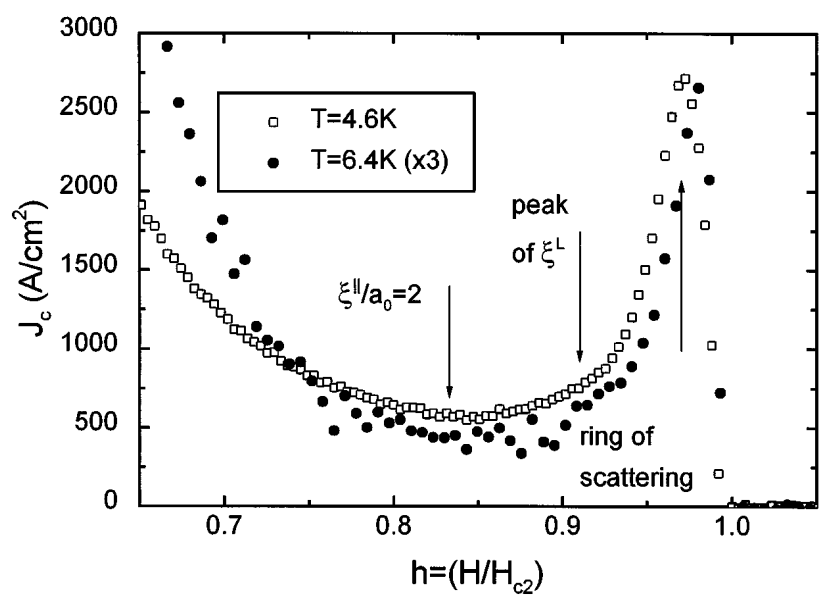

FIG. 4. Shown is the magnetization critical current through the peak effect for $T=4.6 \mathrm{~K}$ (open squares) and $T=6.4 \mathrm{~K}$ (solid circles). The structural regimes are identified by the arrows. 
Combining the transport and SANS data leads to a detailed description for the disordering of the FLL through the peak effect, as sketched in Fig. 4. In general, the positional correlation length is dropping for $h>$ 0.5 . The minimum critical current and the departure of the critical current from the simple constant pinning force, constant $J_{c} h$ limit coincides with $\xi^{\|} / a_{0} \sim 2$. As the critical current starts to rise, ultimately leading to the peak effect, we see three signs of disordering. First, positional order is completely lost, as $\xi^{\|} / a_{0} \rightarrow 1$. Second, there is a rapid reduction of the orientational correlation length $\xi^{\perp} / a_{0}$. Third, there is a peak in the longitudinal correlation length. From this point up to the peak, the FLL is structurally similar to a hexatic of long flux lines as shown in the center panel of Fig. 2, although the orientation is not spontaneous, but rather imposed by the $\mathrm{Nb}$ crystallography. At the location of the peak, $\xi^{\|} / a_{0} \sim 1$, and there is also complete loss of orientational order, with $\xi^{\perp} / a_{0} \sim 1$. The scattering in the plane of the detector is then a uniform ring as shown in the bottom panel of Fig. 2, which has not been previously observed. Structurally, this corresponds to a completely amorphous pattern of lines. Throughout this range, the longitudinal correlation length, $\xi^{L}$, although falling, remains long.

It is tempting to interpret the isotropic ring of scattering at the peak in the critical current as the signature of a pinned liquid of flux lines. A thermodynamic liquid, with zero critical current, is not seen, and would not be expected in our $\mathrm{Nb}$ based on a Lindemann criterion [16] estimate. Further, the region above the peak in $\xi^{L}$ is well described by the amorphous limit of the collective pinning theory, which describes solid vortex phases. We conclude that the amorphous FLL in this regime is a highly disordered solid consisting of linelike objects. However, the static structure factor alone is insufficient to distinguish definitively between a pinned liquid and an amorphous solid. Resolution of this distinction requires dynamic measurements of the structure. One could either try to quench the amorphous system seen close to $H_{c 2}$ in order to estimate the time scales involved or examine the FLL in the presence of a current in excess of the critical current. Although there are as yet no data near the peak effect, both early data [17] in Nb near $h \sim 0.5$, and our studies [9] of $\mathrm{NbSe}_{2}$, also for $h<0.5$, show the dramatic effect a transport current can induce. A pinned liquid should remain disordered above the critical current, whereas an amorphous solid should order into a lattice as seen in low field $\mathrm{NbSe}_{2}$ experiments. Specific predictions for the ordering in the vicinity of the peak effect for the case of $\mathrm{NbSe}_{2}$ have been presented by Henderson et al. [18] based on transport data. No conclusive results for either type of experiment could be obtained with our present setup and samples.

In conclusion, we have combined SANS with thermodynamic and transport data on a high quality $\mathrm{Nb}$ crystal to obtain a complete scenario for the field cooled FLL through the regime of the peak effect. We see a variety of FLL structures as the lattice disorders through hexatic and fully amorphous regimes. We believe these data will be an essential component in the interpretation of this fascinating regime of conventional, type-II superconductors.

*Present address: Biosense Ltd., Tirat HaCarmel, 39101 Israel.

†Present address: Department of Physics, Purdue University, West Lafayette, IN 47907.

[1] R. Merithew et al., Phys. Rev. Lett. 77, 3197 (1996); S. Bhattacharya and M. J. Higgins, Phys. Rev. Lett. 70, 2617 (1993); A.C. Marley, M. J. Higgins, and S. Bhattacharya, Phys. Rev. Lett. 74, 3029 (1995).

[2] P. L. Gammel, D. A. Huse, and D. J. Bishop, in Spin Glasses and Random Fields, edited by P. Young (World Scientific, Singapore, 1997).

[3] G. D’Anna et al., Phys. Rev. Lett. 75, 3521 (1995), and references therein.

[4] D. K. Finnemore et al., Phys. Rev. 149, 231 (1966).

[5] D. K. Christen et al., Phys. Rev. B 21, 102 (1980).

[6] D. K. Christen et al., Phys. Rev. B 15, 4506 (1977).

[7] P. L. Gammel et al., Phys. Rev. Lett. 72, 278 (1994).

[8] J. R. Clem, J. Low Temp. Phys. 18, 427 (1975).

[9] U. Yaron et al., Nature (London) 376, 753 (1995).

[10] G. Lippmann, J. Schelten, and W. Schmatz, Philos. Mag. 33, 475 (1976).

[11] J. W. Lynn et al., Phys. Rev. Lett. 72, 3413 (1994); E. M. Forgan et al., Phys. Rev. Lett. 74, 1697 (1995); J. W. Lynn et al., ibid. 74, 1698 (1995).

[12] For a general review, see G. Blatter et al., Rev. Mod. Phys. 66, 1125 (1994).

[13] U. Yaron et al., Phys. Rev. Lett. 73, 2748 (1994).

[14] P. H. Kes and C. C. Tsuei, Phys. Rev. B 28, 5126 (1983); G. P. van der Meij and P.H. Kes, Phys. Rev. B 29, 6233 (1984); H. R. Kerchner, J. Low Temp. Phys. 50, 337 (1983); E. H. Brandt, Phys. Rev. B 18, 6022 (1978).

[15] J. Mannhart, in Physics of High Temperature Superconductors, edited by S. Maekawa and M. Sato (SpringerVerlag, New York, 1992).

[16] A. Houghton, R. A. Pelcovitz, and A. Sudbo, Phys. Rev. B 40, 6763 (1989).

[17] P. Thorel, R. Kahn, and Y. Simon, J. Phys. 34, 447 (1973); J. Schelten, H. Ullmaier, and G. Lippmann, Phys. Rev. B 12, 1772 (1975).

[18] W. Henderson et al., Phys. Rev. Lett. 77, 2077 (1996). 